\section{Cómo desarrollar ciencia odontológica en el Perú}

\author{
How to develop dental science in \\ Peru
}

Renzo Alberto Ccahuana-Vasquez ${ }^{1, a}$

${ }^{1}$ Oral Care Department, Procter \& Gamble. Kronberg, Germany. a PhD, Clinical Scientist.

\section{Correspondencia:}

Renzo Alberto Ccahuana-Vasquez

Correo electrónico: vasquez.r.15@pg.com

Procter \& Gamble Service GmbH. Frankfurterstrasse 145,61476

Kronberg im Taunus, Germany.

Fecha de recepción: 19/10/18

Fecha de aceptación: 31/10/18
La baja producción científica en salud en las universidades del Perú ha sido materia de diversas discusiones y análisis, habiéndose identificado diversos factores causantes como la indiscriminada aparición de universidades, la mínima inversión del Estado en ciencia y tecnología, la falta de recursos humanos debidamente calificados, la ausencia de laboratorios de investigación entre otros ${ }^{1,2}$.

Las facultades de odontología no han sido ajenas a este problema, y básicamente se han centrado en el entrenamiento de los estudiantes en procedimientos clínicos siguiendo diversas filosofías y metodologías de universidades extranjeras reconocidas, pero no le han dado la misma prioridad al desarrollo de la ciencia que promueva la propia evidencia científica ${ }^{3}$.

A continuación enfatizo algunas sugerencias para la necesaria activación de la producción científica odontológica en el Perú para poder aumentar su competitividad en Latinoamérica.

Estricto control de la calidad universitaria. Hasta la década de los ochenta, las universidades basaban sus objetivos y metas en un contexto local, regional o nacional. La globalización ha establecido nexos de comunicación e interdependencia entre países e instituciones creando la ruptura de las fronteras en todas las áreas de la sociedad. La educación no escapa a este fenómeno, lo que trae como consecuencia la búsqueda de la estandarización de los niveles de calidad a un nivel internacional. En este contexto, se ha evidenciado grandes deficiencias de la educación superior nacional y a partir de esto se ha buscado equiparar y estandarizar infraestructura, métodos de trabajo y personal calificado.

Actualmente se han dado pasos concretos para el licenciamiento de las universidades peruanas a través de la verificación de las condiciones básicas y la autorización de su funcionamiento por medio de la Superintendencia Nacional de Educación Universitaria (SUNEDU) y la acreditación de estas por medio del Sistema Nacional de Evaluación, Acreditación y Certificación de la Calidad Educativa (SINEACE) con el objetivo de garantizar la calidad educativa y normar, en nuestro caso, la habilitación de las facultades de odontología incluyendo sus escuelas de pre y postgrado con la subsecuente fiscalización para mantener niveles mínimos de calidad que brinden al país profesionales bien formados ${ }^{4}$. Se espera que estos organismos incluyan en un futuro cercano los parámetros de calidad de investigación científica por facultades como por ejemplo, el número de artículos que deben ser publicados por año o por trienio y en qué bases de datos. 
Cambio de concepto sobre producción científica. La evaluación de calidad previamente descrita debe envolver no solamente los criterios de entrenamiento de los profesionales sino también la producción de ciencia con estándares internacionales. La investigación como uno de los pilares de la educación universitaria ha sido siempre mencionada en los estatutos universitarios pero su ejecución ha sido pobremente llevada a la práctica. El número de tesis de pregrado y postgrado como también de manuales y libros ha sido considerado por muchas décadas la principal fuente de producción científica en las facultades de odontología en el Perú, de esta forma han podido justificar su papel en la producción de ciencia. Con el proceso de globalización mencionado anteriormente, ha quedado en evidencia que la producción científica es medida primordialmente en número de artículos científicos publicados en revistas indizadas. Considerando que uno de los objetivos de la producción científica es la diseminación de ciencia, las tesis han provocado el estancamiento y la limitación en la difusión de su contenido porque no presentan una información concisa que les permita ser atractivas en estos tiempos dinámicos y porque no son incluidas en las principales bases de datos del mundo. Esto se hace más evidente cuando vemos las estadísticas, por ejemplo los índices de publicación de tesis de las escuelas de pregrado y postgrado de la facultad de odontología de la Universidad Nacional Mayor de San Marcos en el periodo 20052015, mostró que de 269 tesis sustentadas 35 (13\%) fueron publicadas en revistas científicas y ese número cae a $11(4,08 \%)$ cuando se refiere a revistas indizadas ${ }^{5}$, lo cual evidencia que son necesarios cambios para viabilizar sus respectivas publicaciones como artículos.

Escuelas de postgrado como pilares de la producción de ciencia. Las escuelas de postgrado tienen la enorme responsabilidad de brindar los más altos grados académicos: magíster y doctor; los profesionales que opten estos grados deberían formar parte del cuerpo docente universitario y del grupo de investigadores en el país.

Las tesis para alcanzar estos grados deben ser vinculadas a estudios originales, con planes experimentales y datos robustos que garanticen su publicación en revistas indizadas. Estas escuelas deben ser los pilares para concluir el ciclo del desarrollo de la ciencia convirtiendo las tesis en artículos científicos de calidad. Los esfuerzos y los escasos recursos serán malgastados y desaprovechados si los resultados de un estudio no se publican para el conocimiento, análisis, validación o refutación de la comunidad científica ${ }^{5}$.

Incremento del número de revistas odontológicas científicas nacionales. La obligatoriedad de tener una revista de difusión científica indizada debería ser incluido como uno de los requisitos para lograr la acreditación de las facultades de odontología en el Perú, al menos en las que existan cursos de postgrado. Parece incongruente que existan 41 instituciones de enseñanza odontológica ${ }^{6}$ y que únicamente seis revistas odontológicas peruanas estén indizadas en la base de datos LILACS, solo una de ellas indizada en SCIELO y ninguna en bases de datos de nivel mundial como MEDLINE o SCOPUS ${ }^{7}$, demostrando que no es solo un problema de cantidad de publicaciones sino también de calidad.

Inversión del Estado y sus alternativas. Para el desarrollo de la actividad científica definitivamente es necesaria la participación económica del Estado. Ha sido muy discutido el déficit de la inversión del gobierno peruano en la investigación científica y a pesar de que en los últimos años ha habido la intención de estimular la investigación por vía del incremento sustancial del presupuesto nacional para esta área, la disponibilidad de becas para realizar estudios de maestría y doctorado en el extranjero, la presencia de algunos fondos concursables para la realización de proyectos de investigación, estas iniciativas son insuficientes, pues continuamos por debajo de los niveles de inversión gubernamental en ciencia y tecnología de países de nuestra región como Chile y Brasil, que además han iniciado este proceso mucho antes y tienen una presencia de vanguardia en la región. Sin embargo, no solo recae toda la responsabilidad en el Estado, las universidades peruanas han descuidado por mucho tiempo su papel de desarrolladores de ciencia, centrándose, en el caso de las facultades de odontología, en el entrenamiento práctico del dentista pero descuidando su papel en la producción de evidencia científica, que en la ausencia o déficit de laboratorios de investigación, de fondos concursables y de profesores con los debidos títulos y remuneración adecuada que les permita una dedicación exclusiva han llevado a la casi nula participación de nuestro país en el contexto científico odontológico regional y mundial. Ante la ya mencionada insuficiente inversión estatal, las facultades de odontología tienen que establecer otras opciones para financiar proyectos de investigación e infraestructura, como por ejemplo, el establecimiento de redes de trabajo o de colaboración nacional o internacional ${ }^{1}$, facilitar el uso de infraestructura y laboratorios entre universidades, atraer inversiones extranjeras a través de la investigación de productos naturales peruanos que puedan tener uso en salud oral, crear temáticas de impacto mundial poco exploradas que solo pueden ser estudiadas en nuestra región, como por ejemplo, evaluar diferentes variables de salud oral y sistémica en relación con diferentes altitudes geográficas.

Simplificación de procesos. Hay cambios que se pueden dar dentro de las instituciones superiores que pueden facilitar la mejora en la producción científica, como por ejemplo, simplificar la redacción de tesis de los cursos de postgrado como se ha llevado a cabo en varias facultades de odontología del exterior que cambiaron la producción de tesis tradicionales por tesis en formato de artículo científico, como la Universidad de Campinas de Brasil o la Academic Centre for Dentistry Amsterdam (ACTA) en Holanda, lo que ha facilitado a profesores y estudiantes el proceso de redacción y de la publicación científica. Estas mejoras han sido ya utilizadas con éxito también en el Perú en las facultades de medicina de la Universidad Peruana Cayetano Heredia, 
la Universidad Santo Toribio de Mogrovejo y la Universidad Peruana de Ciencias Aplicadas con resultados alentadores por el incremento del número de artículos publicado en revistas indizadas ${ }^{8}$. En los últimos años los centros de postgrados de odontología de las universidades Cayetano Heredia y la Científica del Sur se han adherido a esta propuesta y en los próximos años veremos el real impacto de este cambio en el número de artículos publicados.

Integración de los investigadores nacionales en el extranjero en el contexto nacional. Siendo que los recursos humanos calificados en investigación son limitados en el país, la permanente colaboración de odontólogos investigadores peruanos que se encuentren laborando en el exterior es muy importante. Su participación vía asesoramiento de estudiantes de postgrado, apoyo en la conformación de redes colaborativas, facilitando la firma de convenios institucionales (para que los estudiantes en formación científica puedan realizar parte de sus trabajos en el exterior), formando parte de los cuerpos editoriales de las revistas científicas locales y compartiendo su experiencia en los centros de pregrado y postgrado en el Perú debe ser impulsado.

Los investigadores peruanos en el extranjero tenemos la responsabilidad de formar parte de esta búsqueda de identidad científica nacional y las facultades de odontología juntamente con organismos como el Consejo Nacional de Ciencia, Tecnología e Innovación (CONCYTEC) deberían facilitar el envolvimiento y participación de estos investigadores.

Seguir ejemplos de éxito. La odontología brasileña ha conseguido las últimas décadas un vertiginoso destaque internacional clínico y científico. En una revolución científica que comenzó en la década de los setenta de la mano de sus universidades públicas, Brasil ha trabajado para tener su propia identidad científica y la odontología no ha sido la excepción. Este proceso tuvo como principales factores: la robusta inversión gubernamental en infraestructura y recursos humanos, el establecimiento y constante renovación de laboratorios de investigación, la incesante formación de investigadores de calidad y la alianza con los mejores centros de investigación del mundo. Los resultados de varios años de dedicación nos indican que la producción odontológica brasileña creció de 171 publicaciones en 2002 a 1533 publicaciones en 2012 en la base de datos SCOPUS 9 Asimismo, tienen una numerosa y consistente participación en los principales Congresos Internacionales de Investigación Odontológica, presentan numerosas revistas científicas indizadas en las principales bases de datos regionales y mundiales, numerosas publicaciones en las principales revistas odontológicas del mundo y renombrados investigadores que trascendieron fronteras y que hoy forman parte de los principales centros de investigación del mundo. Todos estos progresos, les han brindado la visibilidad internacional a las principales facultades brasileñas y actualmente son reconocidas en distintos rankings entre las mejores del mundo.

Garantizar la ejecución de un plan a largo plazo. La coyuntura nacional en las últimas décadas ha estado colmada de constantes crisis políticas, sociales y económicas creando ambientes de inestabilidad que tienen un gran impacto en todas las áreas de la sociedad. Se debe crear el compromiso y los mecanismos para garantizar la independencia y autonomía de las instituciones educativas superiores y de los organismos encargados del fomento de la ciencia y tecnología dentro de un plan de activación de la investigación científica. En este sentido, la calidad de la enseñanza universitaria debe ser alta y no debe ser vulnerada por ningún interés político o económico.

Lograr la competitividad de la investigación odontológica peruana es extremamente difícil, pero no imposible. Si bien los recursos de todo tipo son importantes y necesarios, lo más importante es el compromiso de las facultades, sus profesores y sus investigadores para lograr dicho objetivo.

\section{Referencias bibliográficas}

1. Huamaní C, Mayta-Tristán P. Producción científica peruana en medicina y redes de colaboración, análisis del Science Citation Index 2000-2009. Rev Peru Med Exp Salud Publica. 2010;27(3):315-25.

2. Grados-Pomarino S. Problemática de la investigación científica. Odontol Sanmarquina [editorial]. 2018;21(2):73-74.

3. Miyashira J. Publicación científica: Un debe ser de las instituciones de educación superior [editorial]. Rev Med Hered. 2017;28:73-74.

4. Ruiz-Quilcat C, Manrique-Chávez JE. Acreditación universitaria en las carreras de odontología en el Perú. Rev Estomatol Herediana. 2016;26(2):85-91.

5. Castro-Rodriguez Y, Cósar-Quiroz J, Arredondo-Sierralta T, Sihuay-Torres K. Producción científica de tesis sustentadas y publicadas por estudiantes de Odontología. Educ Med 2017. Disponible en: http://dx.doi.org/10.1016/j.edumed.2017.04.002 [en prensa].

6. Colegio Odontológico del Perú [consultado 17 octubre 2018] Disponible en: http://www.cop.org.pe/directorio-de-facultades-de-odontologia.

7. Mattos-Vela MA, Cuadrao Zavaleta L. Seguimos avanzando [editorial]. Odontol Sanmarquina. 2017;20(1):3-4.

8. Mayta-Tristán P. Tesis en formato de artículo científico: oportunidad para incrementar la producción científica universitaria [editorial]. Acta Med Peru. 2016;33(2):95-8.

9. Normando D. The Brazilian dental science [editorial]. Dental Press J Orthod. 2014;19(2):14. 